Article

\title{
Critical Stability of the Negatively Charged Positronium-Like Ions with Yukawa Potentials and Varying $\mathrm{Z}$
}

\author{
Sabyasachi Kar ${ }^{1, *(D)}$, Yu-Shu Wang ${ }^{1}$, Yang Wang ${ }^{1}$ and Yew Kam Ho ${ }^{2}$ \\ 1 Department of Physics, Harbin Institute of Technology, Harbin 150001, China; \\ wangyushu@stu.hit.edu.cn (Y.-S.W.); yangwang0624@foxmail.com (Y.W.) \\ 2 Institute of Atomic and Molecular Sciences, Academia Sinica, Taipei 106, Taiwan; \\ ykho@pub.iams.sinica.edu.tw \\ * Correspondence: skar@hit.edu.cn
}

Received: 29 April 2019; Accepted: 29 May 2019; Published: 3 June 2019

\begin{abstract}
The question of stability of a given quantum system made up of charged particles is of fundamental interest in atomic, molecular, and nuclear physics. In this work, the stability for the negatively charged positronium (Ps)-like ions or the three-body system $\left(\mathrm{Ze}^{+}, e^{-}, e^{-}\right)$with Yukawa potentials is studied using correlated exponential wavefunctions based on the Ritz variational method. We obtained the critical screening parameter $\mu_{C}$ as a function of the continuously varied nuclear charge $Z$, the critical nuclear charge $Z_{C}$ as a function of the screening parameter $\mu$, and the ionization energies in terms of the screening parameter $\mu$ and $Z$. The critical nuclear charge for the bare Coulomb system $\left(\mathrm{Ze}^{+}, e^{-}, e^{-}\right)$obtained using 700 -term correlated exponential wavefunctions is in accord with the reported results. The ionization energy, $\mu_{C}$, and $Z_{C}$ for the Yukawa system $\left(Z e^{+}, e^{-}, e^{-}\right)$exhibit interesting behaviors. The present study describes the possible nonexistence of Borromean binding as well as Efimov states. The possible existence of quasi-bound resonances states for the negatively charged screened Ps-like ions is briefly discussed.
\end{abstract}

Keywords: critical stability; critical nuclear charge; critical screening parameter; Yukawa potentials; Debye potentials; positronium negative ion; exponentially correlated wavefunctions; variational method

\section{Introduction}

Despite the fact that the stability of few-body Coulomb systems is an old topic of research [1-6] and several review articles on this problem are available in the literature [1-15], this problem is still a fascinating topic of research and is of fundamental interest in several areas of physics such as atomic, molecular, and nuclear physics [8-15]. This paper deals with an investigation on the stability of three-body Coulomb systems, made up of two electrons and a particle of charge $Z$ having the mass of a positron, with continuously varying $Z$ and interacting with Yukawa potentials [16] or the Debye potentials [17]. We define such three-body system as $\left(Z e^{+}, e^{-}, e^{-}\right)$. For $Z=1$, the system is known as the positronium (Ps) negative ion. So, the system $\left(\mathrm{Ze}^{+}, e^{-}, e^{-}\right)$can also be considered as the negative Ps ionic systems for continuously varying $Z$ with values less than or equals to 1 . Mills first reported observation for this positronic system in the laboratory [18]. Theoretical studies and experimental activities for the Ps negative ion have been summarized in the recent articles [19-25]. Due to importance of the study of stability for various physical systems, e.g., positronic atoms, antimatter compounds, and charged excitons in semiconductors [7], and due to the importance of the stability of few-charge Coulomb systems with the influence of external environments [26-31], it is relevant to search for the stability of the negatively charged Ps ionic systems with or without influence of external environments. 
The main interest of this work aims to the precise determination of the critical nuclear charge $Z_{C}$ and the critical screening parameter $\mu_{C}$ for the bare Coulomb system $\left(\mathrm{Ze}^{+}, e^{-}, e^{-}\right)$and the Yukawa systems $\left(Z e^{+}, e^{-}, e^{-}\right)$. The critical charge $Z_{C}$ denotes a cut-off for which the system under study does not support any bound state for $Z<Z_{C}$, but supports at least one bound state for $Z \geq Z_{C}$. The critical parameter $\mu_{C}$ also indicates cut-off points those are responsible for the determination of bound states, quasi-bound states [32], or Borromean states [33-35]. Suppose the critical screening $\mu_{C}$ admits two values $\mu_{L}$ (the lower critical screening parameter) and $\mu_{U}$ (the upper critical screening parameter) for a given $Z$, the proposed three-body Yukawa system supports bound states for $\mu_{L} \leq \mu \leq \mu_{U}$, subject to the condition that its two-body subsystem $\left(\mathrm{Ze}^{+}, e^{-}\right.$) (or the Ps like system) is stable for $\mu_{L} \leq \mu \leq \mu_{U}$. Such a system represents the quasi-bound states (i.e., the energy levels lying above the respective two-body subsystem for $\left.\mu \leq \mu_{L}\right)$ subject to the criteria that the system $\left(Z e^{+}, e^{-}\right)$is bound for $\mu \leq \mu_{L}$, and also represents the Borromean states for $\mu \geq \mu_{U}$ if the system $\left(Z e^{+}, e^{-}\right)$is unstable for $\mu \geq \mu_{U}$. It is to be noted that the quasi-bound states indicate the existence of shape resonances [31].

For the bare Coulomb case, the critical nuclear charge for the two-electron $\left(Z, e^{-}, e^{-}\right)$ions has been studied recently using the variational method [10,11,15] and the Lagrange Mesh method [12], among these calculations, the best variational result for $Z_{C}$ has been reported so far by Estienne et al. [10]. Kais and Shi [1] obtained the value $Z_{C}$ for the $\left(\mathrm{Ze}^{+}, e^{-}, e^{-}\right)$ion using the finite-size scaling method for quantum systems based on the variational method. Here we estimate $Z_{C}$ for the $\left(Z e^{+}, e^{-}, e^{-}\right)$ions using correlated exponential wavefunctions. Moini [11] reported the value of $Z_{C}$ for the $\left(Z e^{+}, e^{-}, e^{-}\right)$ions using the Hylleraas-type basis functions. Our prediction is in good agreement with the value reported by Kais and Shi [1]. Except the results of Kais et al. [1] and Moini [11], to the best of our knowledge, there are no other results available in the literature for the values of $Z_{C}$ for the $\left(\mathrm{Ze}^{+}, e^{-}, e^{-}\right)$ions.

The stability of few-body systems with Yukawa potentials or Debye potentials are also of great interest due to its extreme importance in determining several features in atomic, nuclear, and molecular physics, such as the Borromean states, Efimov effects, quasi-bound states, critical screening parameter, critical nuclear charge, etc. It is important to mention here that, despite the same mathematical form, the Yukawa potentials are of interest to the nuclear physicists and the Debye potentials are of interest to the plasma physicists. Recently, Montgomery et al. [14] and Sen et al. [13] studied the critical stability for one- and two-electron Yukawa systems with varying nuclear charge. We also presented the critical nuclear charge and critical screening parameter for the $\left(Z, e^{-}, e^{-}\right)$ions with Yukawa potentials and varying $Z$ [15]. Ho studied the doubly excited resonance states for the $\left(\mathrm{Ze}^{+}, e^{-}, e^{-}\right)$ions with screened Coulomb potentials and varying $Z$ [30]. In this study, we also present the critical nuclear charge as a function of $Z$, the critical nuclear charge as a function of screening parameter, the ionization energy in terms of screening parameter, and $\mathrm{Z}$ for the Yukawa system $\left(\mathrm{Ze}^{+}, e^{-}, e^{-}\right)$with varying $\mathrm{Z}$ using the correlated exponential wavefunctions based on Ritz variational principle. The convergence and stability of the present calculations are examined respectively with increasing number of terms in the wavefunctions and with different choices for nonlinear variational parameters in the wavefunctions. Atomic units (a.u.) are used throughout.

\section{Calculations}

One needs to solve the Schrödinger equation to obtain the ground state energy $E(Z, \mu)$ variationally for the proposed Yukawa atoms. The Hamiltonian $H(Z, \mu)$ and wavefunction $\Psi$ for the Yukawa system $\left(\mathrm{Ze}^{+}, e^{-}, e^{-}\right)$can be written as

$$
\begin{gathered}
H(Z, \mu) \Psi(\mu)=E(Z, \mu) \Psi(\mu) \\
H(Z, \mu)=-\frac{1}{2} \nabla_{1}^{2}-\frac{1}{2} \nabla_{2}^{2}-\frac{1}{2} \nabla_{3}^{2}-Z\left[\frac{\exp \left(-\mu r_{31}\right)}{r_{31}}+\frac{\exp \left(-\mu r_{32}\right)}{r_{32}}\right]+\frac{\exp \left(-\mu r_{21}\right)}{r_{21}} \\
\Psi(\mu)=\left(1+\hat{O}_{12}\right) \sum_{i=1}^{N_{P}} P_{i}(\mu) \exp \left(-\alpha_{i} r_{31}-\beta_{i} r_{32}-\gamma_{i} r_{21}\right)
\end{gathered}
$$


The Hamiltonian $H(Z, \mu)$ and wavefunction $\Psi$ for a one-electron $\left(Z e^{+}, e^{-}\right)$ion are as follows

$$
\begin{gathered}
H(Z, \mu)=-\nabla^{2}-Z \frac{\exp (-\mu r)}{r} \\
\Psi(\mu)=\sum_{i=1}^{N_{Q}} Q_{i}(\mu) r^{i-1} \exp (-\delta r)
\end{gathered}
$$

The parameter $\mu$ (units of $a_{0}^{-1}$ ) is known as the Yukawa parameter or the Debye parameter which has to be set zero for the bare Coulomb systems. $\delta, \alpha_{i}, \beta_{i}$, and $\gamma_{i}$ are the nonlinear variational parameters. We select the parameters $\alpha_{i}, \beta_{i}$, and $\gamma_{i}$ by means of the following pseudorandom relations presented in matrix notation

$$
\left[\begin{array}{lll}
\alpha_{i} & \beta_{i} & \gamma_{i}
\end{array}\right]^{T}=\left[\begin{array}{lll}
\langle k \sqrt{2} / 2\rangle\rangle A & \langle\langle\sqrt{3} / 2\rangle\rangle B & \langle\langle k \sqrt{5} / 2\rangle\rangle C
\end{array}\right]^{T}
$$

where $k=i(i+1)$ and $\delta, A, B$, and $C$ are chosen randomly by the trial and error method. The notation $\langle\langle x\rangle\rangle$ denotes the fractional part of a real number $x . P_{i}(\mu)$ and $Q_{i}(\mu)$ are linear coefficients. and $N_{Q}$ indicates the number of terms in wavefunctions (3) and (5), respectively. $\hat{O}_{12}$ is the permutation operator for the two identical particles 1 and 2 representing the electrons. We calculate the upper bound to the true energy based on the Ritz variational principle.

\section{Results and Discussion}

The ground state energies $E(Z, 0)$ for $Z \geq Z_{C}$ obtained from this calculation for the bare Coulomb systems $\left(Z e^{+}, e^{-}, e^{-}\right)$and $\left(Z e^{+}, e^{-}\right)$are presented in Table 1 . Table 1 also presents the systematic shift in energy eigenvalues with decreasing $Z$ up to the neighborhood of $Z_{C}$. The ground state energy for $Z=1$, that is, for the positronium negative ion is taken from our earlier work [36]. The critical nuclear charge obtained from this calculation is $Z_{C}=0.921802443$ which is in good agreement with the results $Z_{C}=0.92180245$ reported by Kais and Shi [1], and is in agreement up to 5 decimal places with the results $Z_{C}=0.92180704$ reported by Moini [11]. There are no other results in the literature to compare our prediction for the ground state energies for the bare Coulomb system $\left(Z e^{+}, e^{-}, e^{-}\right)$and $\left(Z e^{+}, e^{-}\right)$. However, the ground state energies presented in Table 1 are convergent and stable up to quoted digits.

Table 1. The ground state energies $E(Z, 0)$ for $\left(Z e^{+}, e^{-}, e^{-}\right)$using 700-term exponential basis functions for selected values of $Z$ along with the available data and threshold energy. ${ }^{\text {a }}$ Reference [36].

\begin{tabular}{lll}
\hline \multicolumn{1}{c}{$\boldsymbol{Z}$} & \multicolumn{1}{c}{$\left(\mathrm{Ze}^{+}, e^{-}, e^{-}\right)$} & $\left(\mathrm{Ze}^{+}, e^{-}\right)$ \\
\hline 1.0 & $-0.2620050702325^{\mathrm{a}}$ & -0.2500000000000 \\
0.98 & -0.248636029841 & -0.2401000000000 \\
0.95 & -0.229443708915 & -0.2256250000000 \\
0.93 & -0.21726201089 & -0.2162250000000 \\
0.921803 & -0.21243026065 & -0.2124301927023 \\
0.92180250 & -0.21242996923 & -0.2124299622516 \\
0.92180245 & -0.21242994009 & -0.2124299392065 \\
0.921802445 & -0.21242993719 & -0.2124299369020 \\
0.921802444 & -0.21242993659 & -0.2124299364411 \\
0.921802443 & -0.21242993600 & -0.2124299359802 \\
0.9218024426 & -0.21242993576 & -0.2124299357958 \\
0.9218024425 & -0.21242993571 & -0.2124299357497 \\
0.9218024424 & -0.21242993565 & -0.2124299357037 \\
0.921802442 & -0.21242993543 & -0.2124299355193 \\
0.92180244 & -0.21242993424 & -0.2124299345975 \\
\hline
\end{tabular}

The ground state energies $E(Z, \mu)$ for the Yukawa systems $\left(Z e^{+}, e^{-}, e^{-}\right)$and $\left(Z e^{+}, e^{-}\right)$for different values of $Z$ and $\mu$ are presented in Table S1 of Supplementary Materials. The ionization energies (IEs) 
for different values $Z$ as functions of the screening parameters are presented in Figures 1 and 2. It is evident from the Figures 1 and 2 (and also from Table S1) that the $\mu$ dependence of IEs is non-monotonic. With increasing $\mu$, the IE for each $Z$ starts to increase from zero at a lower critical $\mu\left(=\mu_{C}=\mu_{L}\right)$ up to a maximum value at $\mu=\mu_{\max }$ and then begins to decrease until again approaching zero at an upper critical $\mu\left(=\mu_{C}=\mu_{U}\right)$. As mentioned in Section 1, here the critical $\mu_{C}$ assumes two values, one is the lower critical screening parameter $\mu_{L}$ and other one is upper critical screening parameter $\mu_{U}$. The $\mu_{\max }$ in terms of $Z$ is displayed in Table 2 . The $\mu_{\max }$ increases up to a point between $Z=0.70$ and $Z=0.66$, then starts to decrease with decreasing $Z$. The upper and lower critical values are presented in Figure 3 and Table 1 . The upper and lower critical screening parameters meet at a point around $Z=0.14$. This $Z$ value is the maximum value below which the Yukawa atoms or the Debye atoms do not support any bound state for any given screening strength and is denoted here as the final critical charge $Z_{F C}$.

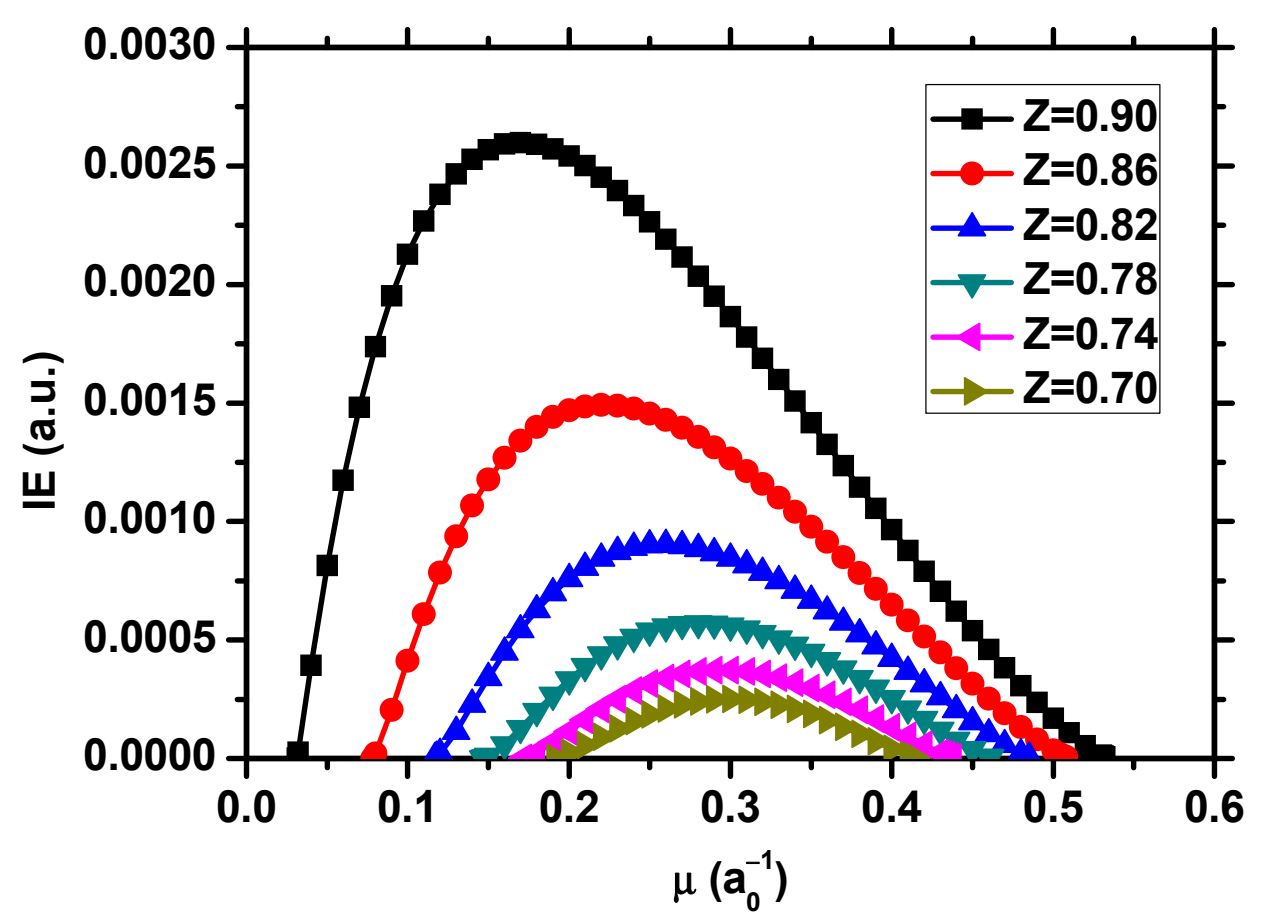

Figure 1. The ground state first ionization energies (IEs) (in atomic units) for six different values of $Z$ in-between 0.70 and 0.90 in 0.04 spacing. 


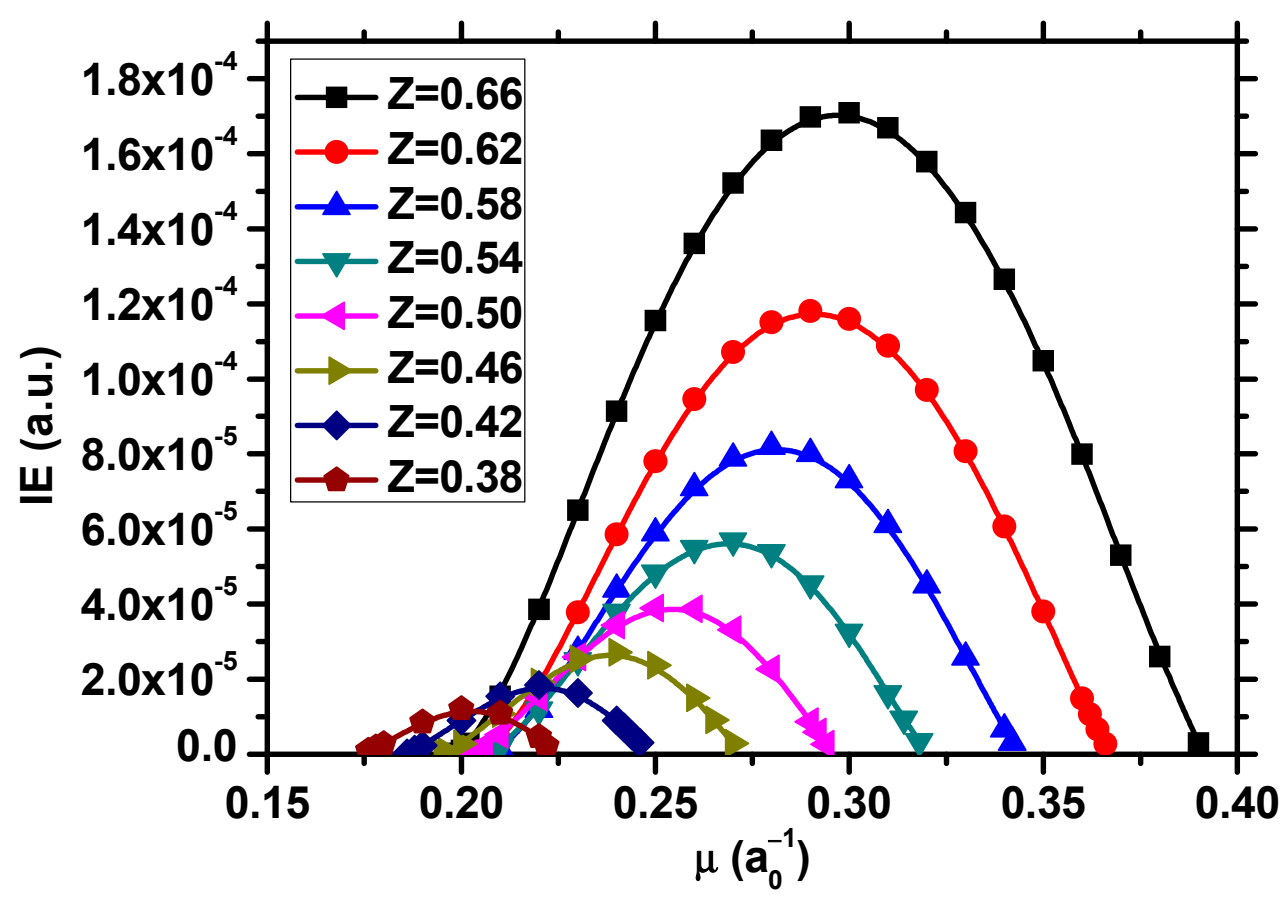

Figure 2. The ground state first ionization energies (IEs) (in atomic units) for eight different values of $Z$ in-between 0.38 and 0.66 in 0.04 spacing.

Table 2. The critical screening parameters for the negatively charged positronium (Ps)-like ions in terms of the nuclear charge. The values of $\mu_{\max }$, the highest peaks of ionization energies (IEs) (also see Figures 1 and 2) as functions with respect to $\mu$ for different $Z$. The three values $Z_{C 1}, Z_{C 2}$, and $Z_{C 3}$ of the critical nuclear charge $Z_{C}$ in terms of $\mu$ for the Yukawa system $\left(Z e^{+}, e^{-}, e^{-}\right)$obtained from the fitting of the values of $\mu_{L}$ and $\mu_{U}$ with a 5 th degree polynomial.

\begin{tabular}{|c|c|c|c|c|c|c|c|}
\hline \multirow{2}{*}{$\mathbf{Z}$} & \multicolumn{2}{|c|}{ Critical Screening Parameter, $\mu_{C}(Z)$} & \multirow{2}{*}{$\frac{\text { Maximum Peak of IEs, } \mu_{\max }(\boldsymbol{Z})}{\mu_{\max }}$} & \multirow{2}{*}{$\mu$} & \multicolumn{3}{|c|}{ Critical Nuclear Charge, $Z_{C}(\mu)$} \\
\hline & Lower $\mu_{L}$ & Upper $\mu_{U}$ & & & $Z_{C 3}$ & $z_{C 2}$ & $z_{C 1}$ \\
\hline 0.14 & 0.078 & 0.078 & 0.078 & 0.00 & 0.921802443 & & \\
\hline 0.18 & 0.096 & 0.103 & 0.10 & 0.05 & 0.88479 & & \\
\hline 0.22 & 0.113 & 0.128 & 0.12 & 0.07798 & 0.86032 & 0.14001 & \\
\hline 0.26 & 0.129 & 0.151 & 0.14 & 0.07815 & 0.86016 & 0.14040 & 0.14001 \\
\hline 0.30 & 0.145 & 0.174 & 0.16 & 0.10 & 0.83862 & 0.18970 & 0.17500 \\
\hline 0.34 & 0.161 & 0.198 & 0.18 & 0.12 & 0.81638 & 0.23648 & 0.20790 \\
\hline 0.38 & 0.174 & 0.222 & 0.20 & 0.15 & 0.77627 & 0.31192 & 0.25823 \\
\hline 0.42 & 0.186 & 0.246 & 0.22 & 0.16 & 0.76018 & 0.33942 & 0.27515 \\
\hline 0.46 & 0.195 & 0.27 & 0.24 & 0.17 & 0.74198 & 0.36885 & 0.29264 \\
\hline 0.50 & 0.202 & 0.294 & 0.25 & 0.18 & 0.72076 & 0.40109 & 0.30905 \\
\hline 0.54 & 0.207 & 0.318 & 0.27 & 0.19 & 0.69466 & 0.44036 & 0.32601 \\
\hline 0.58 & 0.208 & 0.342 & 0.28 & 0.20 & 0.65864 & 0.48474 & 0.34294 \\
\hline 0.62 & 0.206 & 0.366 & 0.29 & 0.205 & 0.63067 & 0.51803 & 0.35140 \\
\hline 0.66 & 0.199 & 0.39 & 0.30 & 0.20865 & 0.57632 & 0.57632 & 0.35757 \\
\hline 0.70 & 0.190 & 0.414 & 0.30 & 0.25 & & & 0.42707 \\
\hline 0.74 & 0.172 & 0.438 & 0.29 & 0.30 & & & 0.51025 \\
\hline 0.78 & 0.147 & 0.461 & 0.28 & 0.35 & & & 0.59307 \\
\hline 0.82 & 0.116 & 0.485 & 0.26 & 0.40 & & & 0.67642 \\
\hline 0.86 & 0.078 & 0.508 & 0.22 & 0.45 & & & 0.76090 \\
\hline \multirow[t]{2}{*}{0.90} & 0.0313 & 0.532 & 0.17 & 0.50 & & & 0.84609 \\
\hline & & & & 0.53198 & & & 0.89999 \\
\hline
\end{tabular}




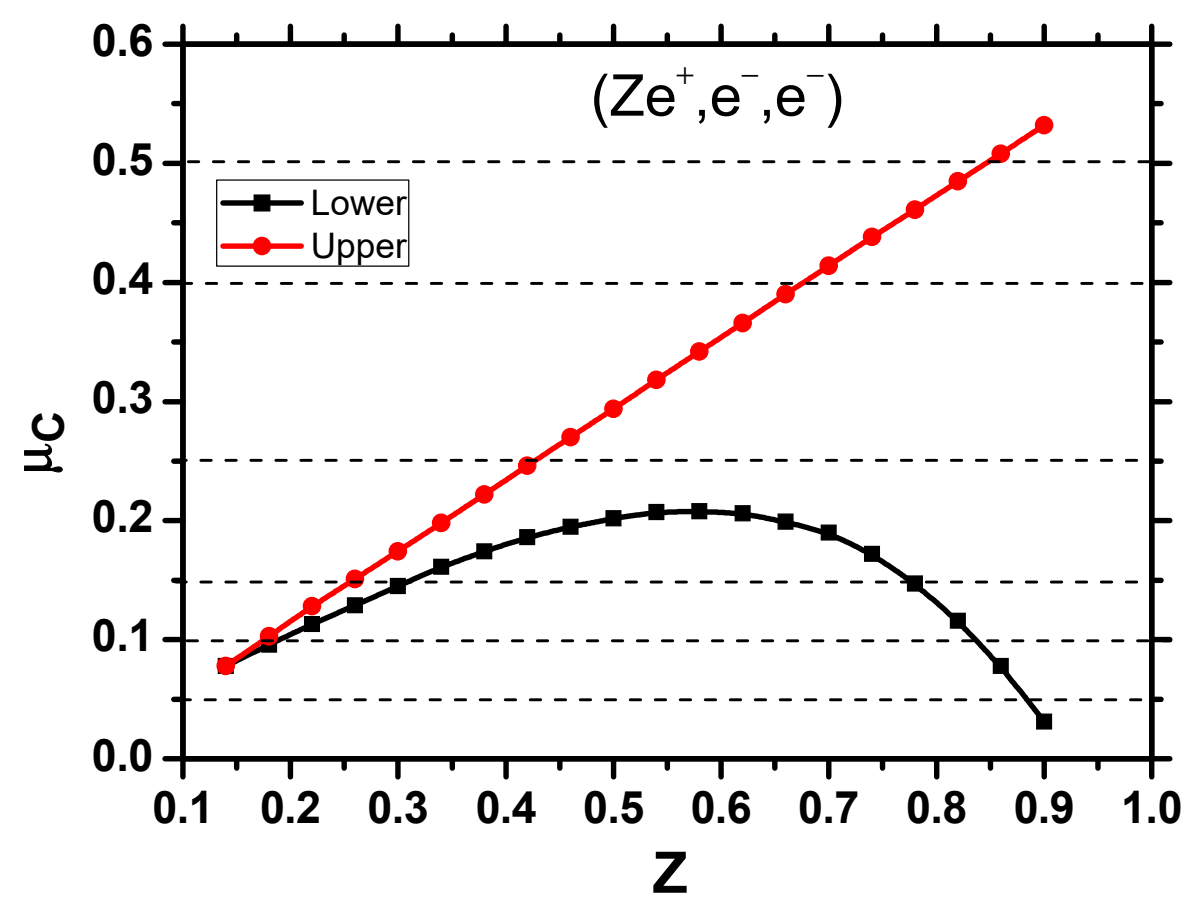

Figure 3. The critical screening parameter versus the nuclear charge for the negatively charged Ps-like ions.

The phenomenon appears in Figures 1 and 2 can be explained from the fact that the binding of an atomic system results from the competition of the Z-dependent attractive potential and Z-independent electron-electron repulsive potential. For the case when the nuclear charge is larger than the critical nuclear charge, the bare Coulomb system is bound as the force due to the attractive potential is stronger than that of the repulsive potential. However, when $Z$ decreases below the critical charge, the three-particle bare Coulomb system becomes unbound as $Z$ affects only the attractive part, and the force due to the repulsive potential hence overtakes the attractive contribution. In the screening environments, the strength of the interaction potential between any given pair of charged particles is to be reduced due to the screening effect. The stability of such screened system or the Yukawa/Debye system depends on the competition between the screened attractive potential and the screened repulsive potential. Though the bare Coulomb system is unbound at certain $Z<Z_{C}$, the Yukawa system is bound for the values of $\mu$ in $\mu_{L} \leq \mu \leq \mu_{U}$. For example, the bare Coulomb system for $Z=0.9$ is unbound, but when the screening effect is increased to $\mu \geq \mu_{L}=0.0313$, the system becomes bound again. This situation suggests that the screening has a stronger effect on the repulsive part than on the attractive part of the potential. As a result, the force due to the screened attractive potential overtakes that of the screened repulsive potential, and the Yukawa system becomes bound with increasing ionization energy as $\mu$ increases. When $\mu$ is increased further up to the value the screening parameter around $\mu=0.17$ (for the case $Z=0.9$ ), the ionization energy starts to decrease with increasing screening parameter as both the attractive and repulsive potentials are reduced moderately, leading to the decrease of the overall ionization energy. When $\mu$ is increased further to the values of screening parameter larger than 0.532 , the upper critical, the screening effect is so strong that the three-particle system becomes unbound again, as demonstrated in Figures 1 and 2 . When $Z$ decreases further, the lower critical screening parameter increases gradually up to the value $Z$ around 0.58 and then begins to decrease until $Z$ approaches $Z_{F C}$. The upper critical screening parameter decreases with decreasing $Z$ until $Z$ approaches $Z_{F C}$. Such phenomena are presented in Figure 3 and such features appear due to the dominant nature of screening effects on attractive potentials, repulsive potential, or overall potentials. The detailed explanation can be well-understood from our previous article [15].

The critical charge $Z_{C}$ for a given $\mu$ is also determined using a polynomial fitting based on the technique prescribed in our recent work [15] and presented here in brief. First, we draw the horizontal 
lines for $\mu$ (say $0.1,0.4,0.5$, etc.) in Figure 3. We estimate the critical $Z$ from the intersections between these horizontal lines and the two critical $\mu_{C}$ curves. For $\mu=0.4$ and 0.5 , the place of intersection is denoted as $Z_{C 1}$ for a given $\mu$. The horizontal lines corresponding to $Z=0.1$ or 0.15 would intersect the two curves at three places, and we named them as (from left to right) $Z_{C 1}, Z_{C 2}$, and $Z_{C 3}$. The horizontal line at $Z=0.05$ crosses the line for critical $\mu_{L}$ curve at $Z_{C 3}$. The values of $Z_{C 1}, Z_{C 2}$, and $Z_{C 3}$ extracted from the 5 th degree polynomial fitting of the values of upper and lower critical screening parameters are listed in Table 1 and depicted in Figure 4. From Figure 4, we can determine what are the values of critical $Z$ for a given $\mu$. For the values of $\mu$ belong to the interval $[0.0,0.14]$, the system is bound for $Z>Z_{C 3}$. For the values of $\mu$ ranging from 0.07815 to 0.20865 , the system is bound when $Z>Z_{C 3}$ or $Z_{C 1}<Z<Z_{C 2}$. For $0.25 \leq \mu \leq 0.532$, the proposed three-body system remains bound for $Z>Z_{C 1}$.

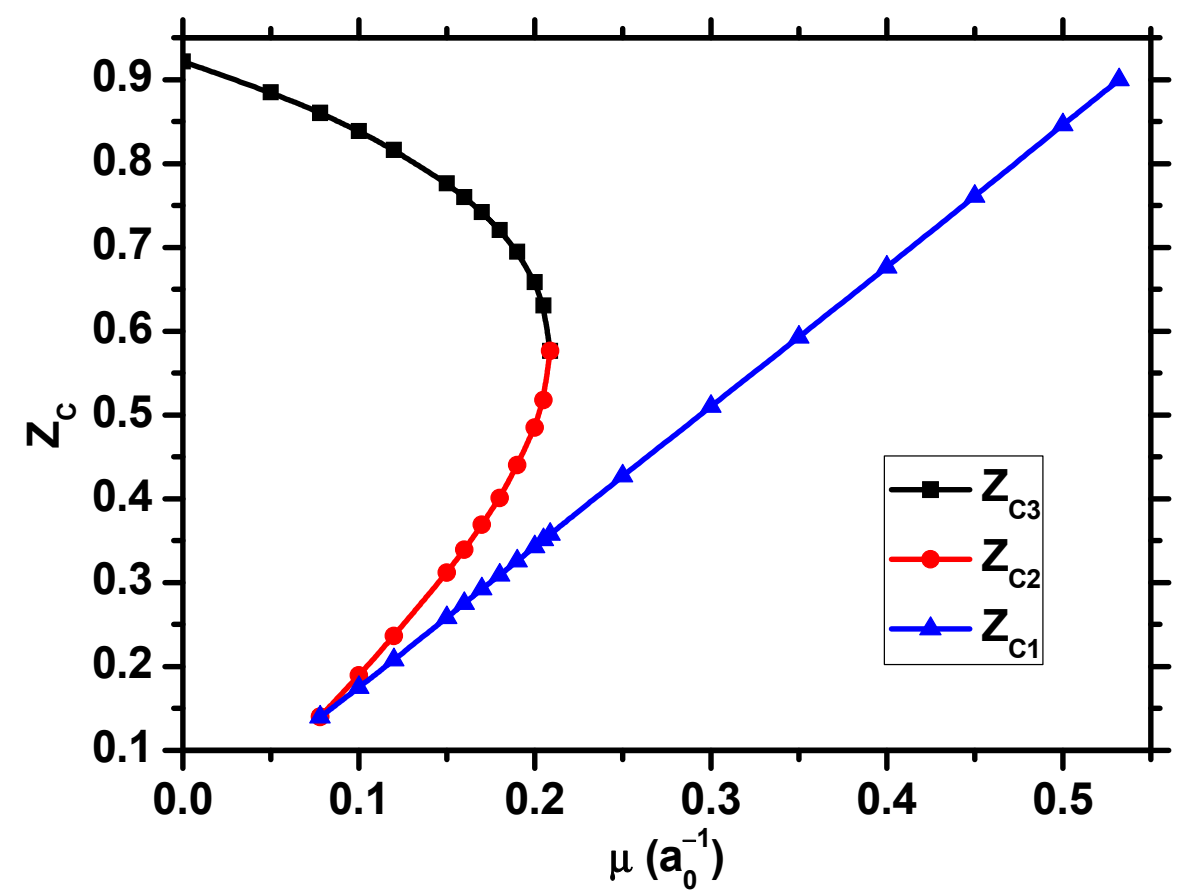

Figure 4. The critical nuclear charge for the negatively charged Ps-like ions as a function of the screening parameter.

One may show interest in the possible phenomena occurred due to the Efimov $[37,38]$ physics (see the review in Reference [35] for details) while studying the bound states for two-body and three-body systems interacting with Yukawa potentials. In this work, it is pertinent to discuss Borromean biding as it is a known fact that the Efimov effect, since its discovery, impinges on several phenomena such as induced long-range interactions, discrete scale invariance, and Borromean binding. As stated earlier in Section 1, basic idea of the three-body Borromean binding states that the three-body Yukawa system can be defined as Borromean when it supports bound states for a fixed range of screening parameters (called the Borromean window) while none of their two-body subsystems are bound in such a range of screening parameter. From the Table S1 (Supplementary Materials), it is clear that the upper critical screening parameter for each $Z$ is similar to the critical screening of the respective two-body subsystem and so from this study, we can only find the range for the Borromean binding $[33,34]$ close to the upper critical screening parameter of the three-body Yukawa system under study. However, the present calculations show that the Borromean window, if it existed for certain Z, would be too narrow and very close to $\mu_{U}$. Table S1 also shows there is no Borromean binding for the region associated with $\mu_{L}$. In other words, $\mu \leq \mu_{L}$, the two-body Yukawa system, supports a bound state while the three-body Yukawa system may support a quasi-bound state, and such a feature does not fall into the Borromean binding criteria. However, as introduced in Section 1, from the quasi-bound 
states, one can determine the shape resonances for the screened positronium negative ionic systems using approaches like the complex-coordinate rotation method [39].

It is also of interest to realize the mass effects on the critical screening. To do so, we compared our results of the critical screening parameters for the negatively charged Ps-like (the system under present study) and the negatively charged H-like ions [15] in Figure 5. Figure 5 indicates that the critical screening parameters decrease to lower values for the decrease of nuclear mass from infinitely heavy to the mass of a positron. However, such a shift to lower values becomes slow with decreasing nuclear charge $Z$.

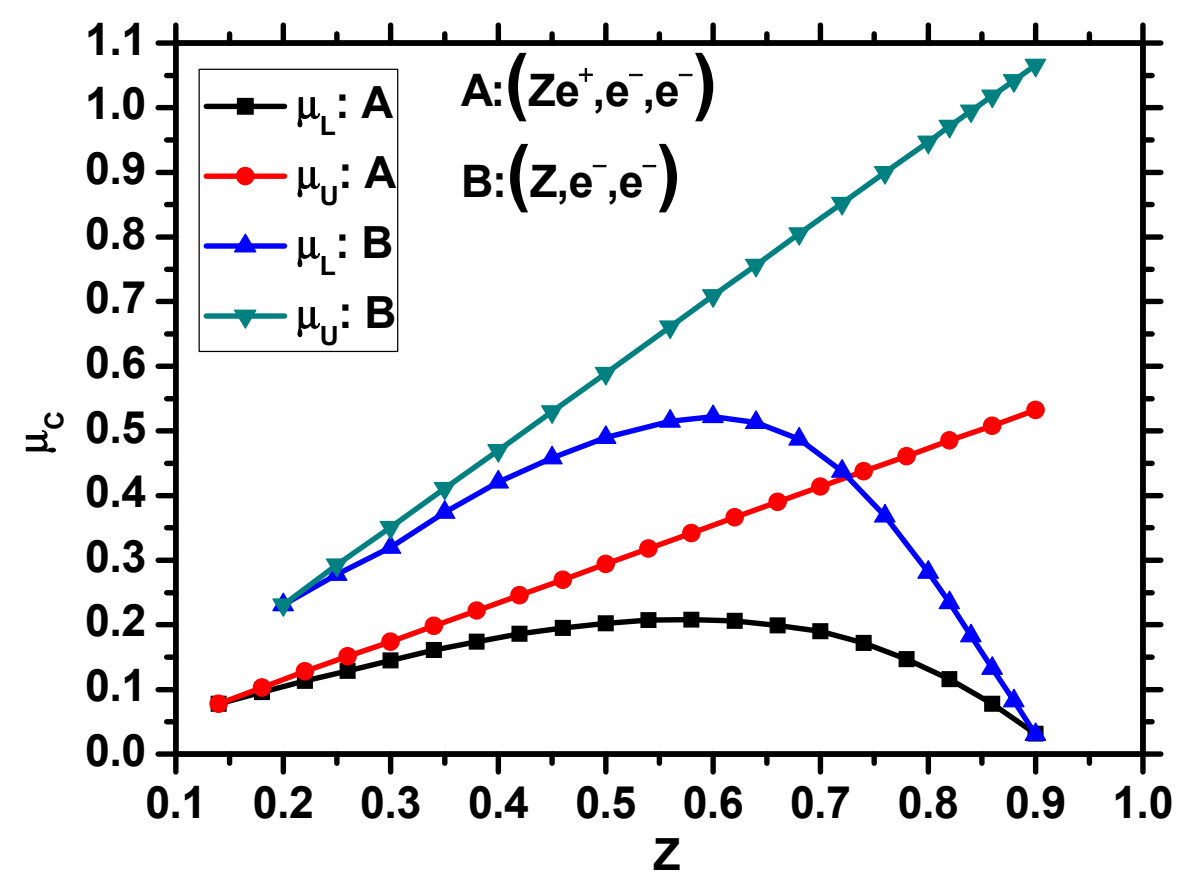

Figure 5. Comparison of critical screening parameters for the negatively charged Ps-like ions and $\mathrm{H}$-like ions in terms of the critical nuclear charge.

Finally, we would like to mention here that the positively charged particle in the present work had values less than unity. As quarks have fractional charges [40], the present work may have relevance in quark-gluon plasma, notwithstanding that under the experimental conditions nowadays, it is unlikely that the present findings would soon be observed in laboratories. We should also mention that fractional charges have been observed in condensed-matter related experiments [41-43], and such fractional charges play an important role in developments on fractional quantum Hall effects [44,45].

\section{Conclusions}

In this work, we have investigated the stability for three-body Yukawa system $\left(Z e^{+}, e^{-}, e^{-}\right)$using correlated exponential wavefunctions based on the Ritz variational method. We have presented the ionization energies in terms of the screening parameter $\mu$ and $Z$, the critical screening parameter $\mu_{C}$ as a function of the continuously varied nuclear charge $Z$, and the critical nuclear charge $Z_{C}$ as a function of the screening parameter $\mu$. The critical nuclear charge for the bare Coulomb system $\left(\mathrm{Ze}^{+}, e^{-}, e^{-}\right)$ obtained using 700-term correlated exponential basis functions is in good agreement with the reported results of Kais and Shi [1]. For the proposed systems, this paper suggests the possible nonexistence of Borromean binding, as well as Efimov states. The possible existence of quasi-bound resonances is also briefly discussed. We hope our findings will be a useful reference for future studies on this topic.

Supplementary Materials: The following are available online at http://www.mdpi.com/2218-2004/7/2/53/s1, for representative data for the ground state energies of the one- and two-electron Yukawa systems. 
Author Contributions: All the authors contributed equally.

Funding: This research received no external funding.

Conflicts of Interest: The authors declare no conflict of interest.

\section{References}

1. Kais, S.; Shi, Q. Quantum criticality and stability of three-body Coulomb systems. Phys. Rev. A 2000, 62, 060502. [CrossRef]

2. Stillinger, F.H. Ground-state energy of two-electron atoms. J. Chem. Phys. 1966, 45, 3623. [CrossRef]

3. Stillinger, F.H.; Stillinger, D.K. Nonlinear variational study of perturbation theory for atoms and ions. Phys. Rev. A 1974, 10, 1109. [CrossRef]

4. Reinhardt, W.P. Dilatation analyticity and the radius of convergence of the $1 / Z$ perturbation expansion: Comment on a conjecture of Stillinger. Phys. Rev. A 1977, 15, 802. [CrossRef]

5. Baker, J.D.; Freund, D.E.; Hill, R.N.; Morgan, J.D. Radius of convergence and analytic behavior of the 1/Z expansion. Phys. Rev. A 1990, 41, 1247. [CrossRef]

6. Ivanov, I.A. References Radius of convergence of the $1 / Z$ expansion for the ground state of a two-electron atom. Phys. Rev. A 1995, 51, 1080. [CrossRef] [PubMed]

7. Armour, E.A.G.; Richard, J.-M.; Varga, K. Stability of few-charge systems in quantum mechanics. Phys. Rep. 2005, 413, 1-90. [CrossRef]

8. Zamastil, J.; Cizek, J.; Skala, L.; Simanek, M. Convergence study of the 1/Z expansion for the energy levels of two-electron atoms. Phys. Rev. A 2010, 81, 032118. [CrossRef]

9. Guevara, N.L.; Turbiner, A.V. Heliumlike and lithiumlike ionic sequences: Critical charges. Phys. Rev. A 2011, 84, 064501. [CrossRef]

10. Estienne, C.S.; Busuttil, M.; Moini, A.; Drake, G.W.F. Critical nuclear charge for two-electron atoms. Phys. Rev. Lett. 2014, 112, 173001. [CrossRef] [PubMed]

11. Moini, A. Critical Nuclear Charge of Quantum Mechanical Three-Body Problem. Electronic Master's Thesis, University of Windsor, Windsor, ON, Canada, 2014.

12. Pilón, H.O.; Turbiner, A.V. Nuclear critical charge for two-electron ion in Lagrange mesh method. Phys. Lett. A 2015, 379, 688. [CrossRef]

13. Sen, K.D.; Katriel, J.; Montgomery, H.E., Jr. A comparative study of two-electron systems with screened Coulomb potentials. Ann. Phys. 2018, 397, 192. [CrossRef]

14. Montgomery, H.E., Jr.; Sen, K.D.; Katriel, J. Critical screening on one- and two-electron Yukawa atoms. Phys. Rev. A 2018, 97, 022503. [CrossRef]

15. Kar, S.; Wang, Y.-S.; Ho, Y.K. Critical stability for the two-electron ions with Yukawa potentials and Varying Z. Phys. Rev. A 2019, 99, 042514. [CrossRef]

16. Yukawa, H. On the interaction of elementary particles. Proc. Phys. Math. Soc. Jpn. 1935, 17, 48-57.

17. Debye, P.; Hückel, E. The theory of electrolytes. I. Lowering of freezing point and related phenomena. Phys. Z. 1923, 24, 185-206.

18. Mills, A.P., Jr. Observation of the positronium negative ion. Phys. Rev. Lett. 1981, 46, 717. [CrossRef]

19. Ho, Y.K. Atomic resonances involving positrons. Nucl. Instr. Meth. Phys. Res. B 2008, 266, 516. [CrossRef]

20. Nagashima, Y. Experiments on positronium negative ions. Phys. Rept. 2014, 545, 95. [CrossRef]

21. Kar, S.; Ho, Y.K. Excitons and the positronium negative ion: Comparison in spectroscopic properties. In Excitons; Pyshkin, S.L., Ed.; INTECH: London, UK, 2018; Chapter 5; pp. 69-90.

22. Kar, S.; Ho, Y.K. Two-photon double-electron D-wave resonant excitation in the positronium negative ion. Euro. Phys. J. D 2018, 72, 193. [CrossRef]

23. Michishio, K.; Kanai, T.; Kuma, S.; Azuma, T.; Wada, K.; Mochizuki, I.; Hyodo, T.; Yagishita, A.; Nagashima, Y. Observation of a shape resonance of the positronium negative ion. Nat. Commun. 2016, 7, 11060. [CrossRef]

24. Cassidy, D.B. Experimental progress in positronium laser physics. Eur. Phys. J. D 2018, 72, 53. [CrossRef]

25. Kar, S.; Wang, Y.-S.; Wang, Y.; Ho, Y.K. Polarizability of negatively charged helium-like ions interacting with Coulomb and screened Coulomb potentials. Int. J. Quantum Chem. 2018, 118, e25515. [CrossRef]

26. Sil, A.N.; Canuto, S.; Mukherjee, P.K. Spectroscopy of Confined Atomic Systems: Effect of Plasma. Adv. Quantum. Chem. 2009, 58, 115. 
27. Kar, S.; Ho, Y.K. Effect of screened Coulomb potentials on the resonance states of two-electron highly stripped atoms using the stabilization method. J. Phys. B 2009, 42, 044007. [CrossRef]

28. Janev, R.K.; Zhang, S.; Wang, J. Review of quantum collision dynamics in Debye plasmas. Matter Radiat. Extrem. 2016, 1, 23. [CrossRef]

29. Kar, S.; Wang, Y.S.; Jiang, Z.; Wang, Y.; Ho, Y.K. Potential-screening on atomic wavelengths. Chin. J. Phys. 2018, 56, 3085. [CrossRef]

30. Ho, Y.K. The Combined Screened Coulomb and Varying Charge Effects on Doubly Excited Resonance States in the Positronium Negative Ion. JPS Conf. Proc. 2017, 18, 011027.

31. Katriel, J.; Montgomery, H.E., Jr.; Sen, K.D. Hund's rule in the $(1 s 2 s)^{1,3} S$ states of the two-electron Debye atom. Phys. Plasmas 2018, 25, 092111. [CrossRef]

32. Dubau, J.; Ivanov, I.A. Numerical calculation of the complex energy of the $1 \mathrm{~s}^{2}$ resonance of a two-electron atom with nuclear charge below the threshold value. J. Phys. B 1998, 31, 3335-3344. [CrossRef]

33. Kar, S.; Ho, Y.K. Borromean windows for $\mathrm{H}_{2+}$ with screened Coulomb potentials. Phys. Rev. A 2012, 506, 282. [CrossRef]

34. Dutta, S.; Saha, J.K.; Bhattacharyya, S.; Mukherjee, P.K.; Mukherjee, T.K. Exotic systems under screened Coulomb interactions: A study on Borromean windows. Phys. Scr. 2014, 89, 015401. [CrossRef]

35. Naidon, P.; Endo, S. Efimov physics: A review. Rep. Prog. Phys. 2017, 80, 056001. [CrossRef]

36. Kar, S.; Ho, Y.K. Positron annihilation in the dense-plasma-embedded Ps ${ }^{-}$. Chem. Phys. Lett. 2006, 424, 403. [CrossRef]

37. Efimov, V. Energy levels arising from resonant two-body forces in a three-body system. Phys. Lett. B 1970, $33,563$. [CrossRef]

38. Efimov, V. Weakly-bound states of three-resonantly interacting particles. Sov. J. Nucl. Phys. 1970, 12, 589.

39. Ho, Y.K. The method of complex coordinate rotation and its applications to atomic collision processes. Phys. Rep. 1983, 99, 1. [CrossRef]

40. Mann, R. An Introduction to Particle Physics and the Standard Model; CRC Press: Boca Raton, FL, USA, 2009; 614p.

41. De-Picciotto, R.; Reznikov, M.; Heiblum, M.; Umansky, V. Direct observation of a fractional charge. Nature 1997, 389, 182. [CrossRef]

42. Saminadayar, L.; Glattli, D.C.; Jin, Y.; Eitenne, B. Observation of the e/3 fractionally charged Laughlin quasiparticle. Phys. Rev. Lett. 1997, 79, 2526. [CrossRef]

43. Martin, J.; Ilani, S.; Verdene, B.; Smet, J.; Umansky, V.; Mahalu, D.; Schuh, D.; Abstreiter, G.; Yacoby, A. Localization of fractionally charged quasi-particles. Science 2004, 305, 980. [CrossRef]

44. Laughlin, R.B. Anomalous quantum Hall effect: An incompressible quantum fluid with fractionally charged excitations. Phys. Rev. Lett. 1983, 50, 1395. [CrossRef]

45. Tsui, D.C.; Stormer, H.L.; Gossard, A.C. Two-dimensional magnetotransport in the extreme quantum limit. Phys. Rev. Lett. 1983, 48, 1559. [CrossRef] 